\section{Payment links now available}

Payment links are now available within Dentally, to support your return to practice as you begin to see patients in person (as and when conditions allow), encouraging social distancing by limiting contact with your reception team.

To help you and your team run a contactless reception, once the dental practitioner has said goodbye to the patient and they have left the surgery rather than a member of the staff having to have physical contact with the patient to take payment, it can now be done securely through an SMS sent straight to the patient.

You can send the payment link as soon as the patient has left the surgery or at certain times during the day as a bulk exercise, it is entirely up to you.

As you would expect from Dentally, this new functionality is easy to set up for your practice. Firstly, set up a Stripe account for the payment (or login to your existing one if you have one) which you can do in the Settings in Dentally. For details on Stripe, visit https://stripe. $\mathrm{com} / \mathrm{gb} /$ pricing. This provides you with a payment screen - which can be customised to include your practice logo and details. The payment page is fully responsive and will work for whatever phone, iPad or device the patient is using.

You can then create a SMS message with the information you wish to include and a data tag for the secure link to your payment page.

Once it is sent to your patient, they see a secure link that allows the patient to pay their bill by clicking on the link to your new payment page, and making a payment using a credit/debit card, Apple Pay, etc. All of the relevant information, such as their name and the payment due, is shown on the payment page. Once completed, the payment will update the patient record within Dentally.

This new feature within Dentally will limit the number of staff members the patient has to come into contact with during their visit to your practice for dental care, but helps you retain a seamless patient experience.

For more information visit: https:// dentally.co/ or call 02038565610.

\title{
For a sustainable future
}

The TePe GOOD range is a unique collection of products with the same TePe quality and design as always. Made from plant-based plastic using renewable plant-based raw materials. New to the TePe GOOD range are the TePe GOOD Mini Flosser and TePe GOOD Tongue Cleaner in pink. Both have been designed in collaboration with dental experts.

The TePe GOOD Mini Flosser is suitable for patients with narrow gaps between the teeth. The handle is made from wood fibres and sugar cane with a floss that is strong, thin and Teflon free. It is available in a 36-pack sustainable bag.

Also joining the range, the TePe GOOD Tongue Cleaner is designed to remove bacteria on the tongue to improve oral health and help prevent halitosis. With three cleaning surfaces for a triple effect, the TePe GOOD tongue cleaner removes bacteria with one stroke.

That's good - for your patients and the planet!

www.tepe.com/uk.

\section{Online training and CPD}

Denplan, part of Simplyhealth, has been adapting and changing to the needs of customers, colleagues, dentists and practices during this unprecedented time, through online training via the Denplan Academy.

With dental practices around the country closing their doors overnight due to COVID-19, the Denplan Academy has adapted from face-to-face training to online sessions for dentists and practice staff.

From re-writing and re-formatting training materials, to re-branding PowerPoints for the most popular 15 courses, the Denplan Academy team is now confidently delivering sessions independently online, continuing to provide personalised learning for dental colleagues.

With online functionality checked and the team confident they could deliver this new way of learning, 20 online sessions have now been booked in and 180 practice team members have now attended one of the sessions. Popular courses include:

- Caring for your patients with dementia: How can we assist patients, as well as people in your local community to live well with dementia

- Complaints handling: Explores methods and techniques to manage patient complaints and review procedures in line with GDC standards

- ECPD and reflective learning: Requirements of the GDC's Enhanced
Continuing Professional Development and how the team can approach the guidelines of Plan, Do, Reflect, Record

- Preparing for your CQC inspection: Examines the process of a CQC inspection and what elements are included in the five fundamental standards of care.

With over 70,000 registrations to date, Denplan's series of clinical webinars ensures practices and teams continue to be supported and can access CPD.

For more information about the Denplan Academy online training, visit https://events.simplyhealth.co.uk/TMT.

Denplan has also announced that it is investing $£ 2$ million to provide essential, fully-certified, personal protective equipment (PPE) to help its Denplan member dentists get safely back to work and protect and care for their patients.

Denplan is currently in final stage discussions with dental suppliers in the UK to create a robust operational process to provide essential PPE equipment to their member practices. Qualifying Denplan member practices will be allocated a fund, based on their Denplan patient numbers, which they can use to order vital PPE equipment directly from the chosen supplier. This fund will allow Denplan practices to select from a range of PPE items to help ensure the safety of their patients and of Denplan dental teams. 